\title{
Renormalization Group Flows of Hamiltonians Using Tensor Networks
}

\author{
M. Bal, ${ }^{1}$ M. Mariën, ${ }^{1}$ J. Haegeman, ${ }^{1}$ and F. Verstraete ${ }^{1,2}$ \\ ${ }^{1}$ Department of Physics and Astronomy, Ghent University, Krijgslaan 281, S9, B-9000 Ghent, Belgium \\ ${ }^{2}$ Vienna Center for Quantum Technology, University of Vienna, Boltzmanngasse 5, 1090 Vienna, Austria
}

(Received 7 April 2017; published 20 June 2017)

\begin{abstract}
A renormalization group flow of Hamiltonians for two-dimensional classical partition functions is constructed using tensor networks. Similar to tensor network renormalization [G. Evenbly and G. Vidal, Phys. Rev. Lett. 115, 180405 (2015); S. Yang, Z.-C. Gu, and X.-G. Wen, Phys. Rev. Lett. 118, 110504 (2017)], we obtain approximate fixed point tensor networks at criticality. Our formalism, however, preserves positivity of the tensors at every step and hence yields an interpretation in terms of Hamiltonian flows. We emphasize that the key difference between tensor network approaches and Kadanoff's spin blocking method can be understood in terms of a change of the local basis at every decimation step, a property which is crucial to overcome the area law of mutual information. We derive algebraic relations for fixed point tensors, calculate critical exponents, and benchmark our method on the Ising model and the six-vertex model.
\end{abstract}

DOI: 10.1103/PhysRevLett.118.250602

Introduction.-The study of phase transitions and critical properties of lattice models has long been at the center of statistical physics. Universal properties of critical systems can be captured by conformal field theories (CFTs), which act as low-energy effective descriptions of critical models and whose scaling dimensions can be related to the critical exponents of asymptotic correlation functions. One way to gain insight into these phenomena is through real-space renormalization group (RG) methods, which predate the development of the modern renormalization group and can be traced back to Kadanoff's block-spin procedure [1]. In his treatment of block-spin methods on the lattice, Wilson emphasized that one should be able to do precise numerical calculations using pure RG methods combined with approximations based only on locality [2]. For real-space RG to work, the effective Hamiltonian at every step should be dominated by short-range interactions as interactions of arbitrary complexity are generated in subsequent iterations. Additionally, the calculation of any particular term in the coarse-grained Hamiltonian should involve but a small number of fine-grained spins.

Tensor networks are efficient, local, real-space variational ansätze for many-body wave functions, which are constructed by mimicking the spatial distribution of entanglement and correlations. Renormalization group methods based on tensor networks satisfy Wilson's requirements insofar as their inherent real-space locality and finite bond dimension restrict the range of newly generated effective interactions and provide a controlled approximation that can be systematically improved.

For two-dimensional lattice systems, the tensor renormalization group (TRG) algorithm [3,4] puts the idea of tensor network renormalization (TNR) into practice in a most explicit way. Wholly based on truncation using singular value decomposition (SVD), this algorithm works extremely well for gapped systems because of the same entanglement reasons that explain the success of the density matrix renormalization group for quantum spin chains [3]. Despite remarkable accuracy in determining critical exponents for finite systems, none of the methods based on the TRG [5-7] is sustainable in the sense that it is capable of yielding true (approximate) fixed points tensors at criticality [8]. Recently, novel TNR algorithms respectively based on the multiscale entanglement renormalization ansatz (MERA-TNR) [8-11] and matrix product states (LoopTNR) [12] have been developed which do manage to flow to approximate fixed point tensors, even at criticality. Our work has been inspired by the latter proposal which formulates TNR in terms of periodic matrix product states (MPSs). For the 2D classical Ising model, impressive numerical results have been obtained that seem to defy the breakdown of the TRG at criticality.

In this Letter, we demonstrate how tensor networks can be used to achieve explicit real-space RG flows in the space of classical Hamiltonians. To this end, we have developed a sustainable and manifestly non-negative TNR method $\left(\mathrm{TNR}_{+}\right)$to coarse-grain classical partition functions. By virtue of the elementwise non-negativity of all tensors involved, we can explicitly associate a Hamiltonian to the fixed point tensors of the RG flow generated by our algorithm. We thus believe our work opens up the possibility to begin to address one of the main concerns raised by the traditional real-space RG community about all TNR schemes: the lack of an insightful RG interpretation of what are essentially supposed to be real-space RG methods [13].

Tensor network renormalization.-The salient features shared by all TNR algorithms developed up to now are twofold. First, the breaking apart of the tensor product structure, which was introduced in the TRG by splitting tensors using SVD, is crucial to the construction of new 
effective degrees of freedom and the removal of correlations. The reason why Kadanoff's spin blocking fails can be traced back to the bounds on correlations imposed by the mutual information between a block and its environment. In order to overcome this barrier, it is essential to reorganize degrees of freedom by doing a local basis transformation. Second, both MERA-TNR and Loop-TNR address the additional need to extend the domain of the coarse-graining step to act on a block of sites in order to remove intrablock correlations. The disentangling power of both MERA-TNR and Loop-TNR can be found in surrounding a block of sites with a coarse-graining operator. This explains, for instance, why there is no way for the TRG, which acts locally on each site, to detect the short-range correlations that it sets out to remove at criticality.

Coarse-graining nonnegative tensor networks.Consider a two-dimensional bipartite square lattice of $N$ classical spins $\left\{s_{i}\right\}$ described by an energy functional $H\left(s_{1}, s_{2}, \ldots\right)$. The classical statistical partition function is then given by

$$
\mathcal{Z}=e^{\beta F}=\operatorname{Tr}_{\left\{s_{1}, s_{2}, \ldots\right\}} e^{\beta H\left(s_{1}, s_{2}, \ldots\right)},
$$

where $F=E-T S$ denotes the free energy. If we imagine the spins living on the vertices of the lattice, the Boltzmann weight of a site depends on the configuration of the bonds connected to the site. We can write these probabilities as a rank-four tensor $A_{i j k l}$, so that the sum over all configurations in the partition function boils down to contracting a non-negative tensor network,

$$
\mathcal{Z}[A]=t \operatorname{Tr} \otimes A_{i j k l} .
$$

By coarse-graining tensor networks, we then refer to a realspace RG procedure constructing a sequence of partition functions $\mathcal{Z}\left[A^{0}\right] \rightarrow \mathcal{Z}\left[A^{1}\right] \rightarrow \cdots \rightarrow \mathcal{Z}\left[A^{s}\right]$, where each effective partition function is defined on a coarser lattice than the one before, until we are left with a single effective site after $s \approx \log _{2}(N)$ iterations. If we now want to additionally retain elementwise non-negativity of all involved tensors at every step, we cannot resort to using SVD, which is the backbone of all other TNR approaches. Instead, we are led to non-negative matrix factorization algorithms [15] to approximate the following matrix factorization problem: Given an elementwise non-negative matrix $A \in \mathbb{R}_{+}^{m \times n}$ and a rank $k \leq \min (m, n)$, find the matrices $X \in \mathbb{R}_{+}^{m \times k}$ and $Y \in$ $\mathbb{R}_{+}^{k \times n}$ minimizing $\|A-X Y\|_{F}^{2}$ [27].

Now let us focus on a block of four adjacent sites [Fig. 1(a)], which we, following Yang, Gu, and Wen [12], interpret as a periodic four-site MPS with respective physical and virtual dimensions. The local coarse-graining procedure then proceeds according to the canonical realspace RG steps by (i) introducing new effective degrees of freedom, which here involves approximating the local block with an ansatz that has a different tensor product

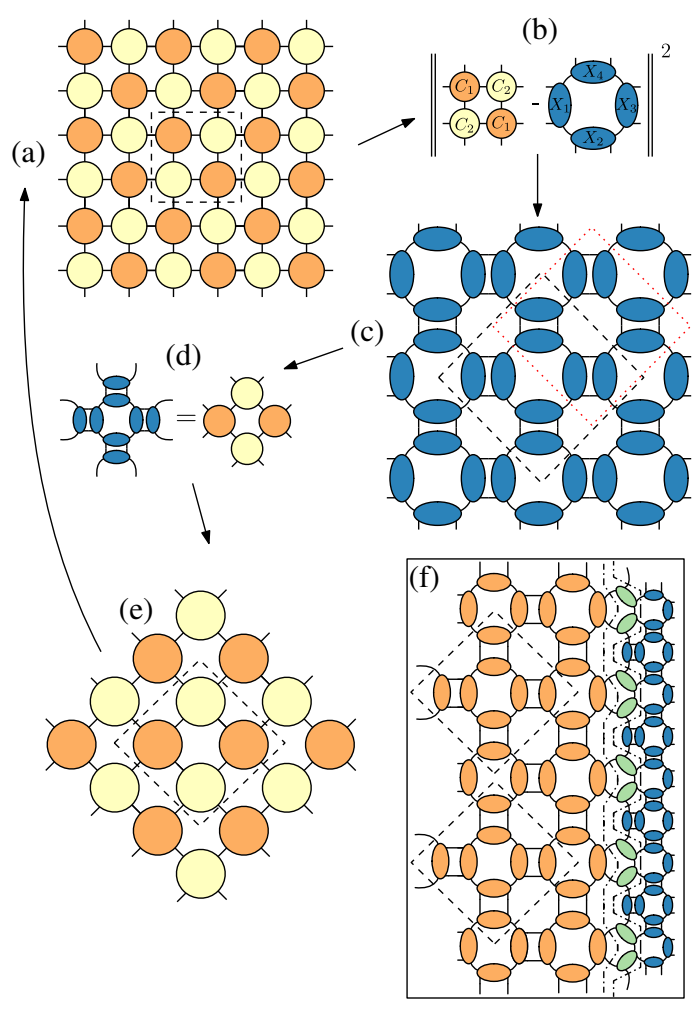

FIG. 1. (a)-(e) Illustration of a single step of the $\mathrm{TNR}_{+}$ algorithm. (a) Starting from a bipartite square lattice, (b) we approximate the periodic MPS representing a block of four sites by a rotated version (c) with a different tensor product structure and (d) contract these numerically optimized tensors exactly to (e) arrive at a coarse-grained tilted lattice. (f) Iterating the $\mathrm{TNR}_{+}$procedure in the presence of an open boundary generates a stochastic MERA.

structure in order to remove short-range correlations [Fig. 1(b)], and (ii) summing over old degrees of freedom by recombining the optimized tensors into new coarsegrained tensors $C_{1}$ and $C_{2}$ [Fig. 1(d)]. The virtual dimension in step (i) can be increased at will, which in turn determines the local dimension of the degrees of freedom living on the new lattice. While step (ii) explicitly sums over the old outer (physical) degrees of freedom to construct the coarse-grained tensors, step (i) also contains an implicit summation over the old inner (virtual) degrees of freedom. After a single RG step, the roles of the physical and virtual MPS dimensions have interchanged and the linear dimension of the lattice is reduced by $\sqrt{2}$. The tensors in Fig. 1(e) then serve as input to the next step, where we take into account that we have to break up the tensor product structure again. Notice that in Fig. 1(c) we identify the coarse-grained lattice with the "vertex" configuration inside the dashed bounding box and not the "plaquette" configuration inside the dotted one. Even though a priori they look similar, the latter configuration leads to worse numerics which can be understood by it not being able to remove short-range correlations of the corner double-line form [15]. 
Renormalization group flow.-In Fig. 1(f), we have depicted the tensor network generated by the action of $\mathrm{TNR}_{+}$on an open boundary of the lattice. In much the same way as the TRG produces a tree tensor network and MERATNR a multiscale entanglement renormalization ansatz [9], our $\mathrm{TNR}_{+}$algorithm builds up a non-negative tensor network approximation to the leading eigenvector of the transfer matrix. Given the non-negativity and the alternating pattern of one iteration "disentangling" (blue tensors) and the next one reducing the degrees of freedom (green tensors), $\mathrm{TNR}_{+}$can be said to generate a stochastic MERA [28]. If we instead track the action of $\mathrm{TNR}_{+}$around an open impurity, we end up with the following MPO after two iterations [15]:

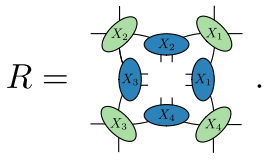

In the scale-invariant regime of the RG flow, this MPO is identified with the radial transfer matrix [9], which can be diagonalized to give $R=\sum_{\alpha} 2^{-\Delta_{\alpha}}|\alpha\rangle\langle\alpha|$. Here, the scaling dimensions $\Delta_{\alpha}$ and approximate lattice representations $|\alpha\rangle$ of the primary fields and descendants of the underlying CFT description are found only if the relative gauge freedom of the coarse-grained partition functions has been fixed, i.e., if the degrees of freedom we deem equivalent after two iterations do indeed match [15]. For critical systems, we thus end up with a window of an approximately invariant alternating sequence of partition functions $\mathcal{Z}\left[C_{1, A}^{*}, C_{2, A}^{*}\right] \rightarrow$ $\mathcal{Z}\left[C_{1, B}^{*}, C_{2, B}^{*}\right]$ after the initial part of the flow has sufficiently suppressed irrelevant lattice details and up until the accumulated truncation errors eventually prevail.

We can furthermore consider the fixed point equations of $\mathrm{TNR}_{+}$as an algebraic set of equations in their own right by finding tensors which (approximately) satisfy
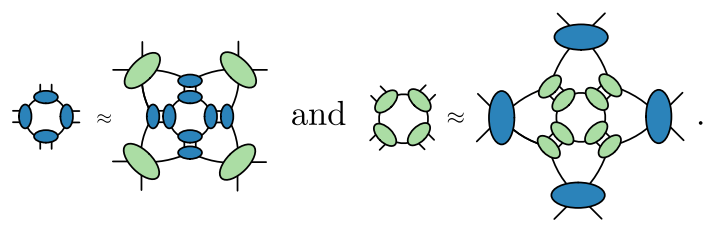

Exact solutions of these equations include trivial product states and Greenberger-Horne-Zeilinger states corresponding to gapped infrared fixed points, potentially with symmetry breaking. Including additional symmetry constraints, there might exist nontrivial solutions which approximately yet accurately satisfy the RG fixed point equations. The sets of these solutions and their stability under perturbations could then point towards the conditions required for a classification of all possible (approximate) RG fixed points of TNR schemes [30,31].

Application to classical partition functions.-We have benchmarked our algorithm on the classical Ising model and the six-vertex model. The partition function of the ferromagnetic Ising model can be encoded by associating a tensor $A_{i j k l}=\sum_{s}(\sqrt{a})_{i s}(\sqrt{a})_{j s}(\sqrt{a})_{k s}(\sqrt{a})_{l s}$ to each vertex, where $a_{m n}=\left[e^{\beta} \mathbb{1}+e^{-\beta} X\right]_{m n}$ denotes the contribution of the interaction between spins $m$ and $n$. The Ising model exhibits a phase transition at the critical temperature $T_{c}=$ $2 / \ln (1+\sqrt{2})$ described by a free fermion $c=1 / 2$ CFT, separating the $Z_{2}$ symmetry-breaking phase for $T<T_{c}$ from a trivial disordered phase for $T>T_{c}$. The partition function of the zero-field six-vertex model can be written in terms of the nonvanishing tensor elements $A_{1111}=A_{2222}=a$, $A_{2112}=A_{1222}=b$, and $A_{2121}=A_{1212}=c$, where $a, b, c$ denote the Boltzmann weights of the allowed bond configurations. In terms of the parameter $\Delta=\left(a^{2}+b^{2}-c^{2}\right) /(2 a b)$, the six-vertex model has a phase boundary determined by $|\Delta|=1$

which separates four phases: two ferroelectric phases for $\Delta>1$, an antiferroelectric phase for $\Delta<-1$, and a gapless disordered phase for $-1<\Delta<1$. The six-vertex model belongs to special classes of Hamiltonians which violate the universality hypothesis in that its phase diagram contains a continuum of critical points with continuously varying critical exponents captured by a free boson $c=1$ CFT. In what follows, we will consider the example of spin ice, i.e., $a=b=c=1$ and $\Delta=0.5$.

In Fig. 2(a), the relative error of the free energy per site $f=-\log (Z) / N$ is plotted at criticality in function of the bond dimension. We observe very accurate free energies, with the difference in accuracy between the simulations of the two models reflecting the less trivial nature of the sixvertex model. To study the implicit approximate scale invariance of the RG flow, we calculate the smallest scaling dimensions from the linear transfer matrix MPO constructed from $4 \times 2$ effective partition function tensors,

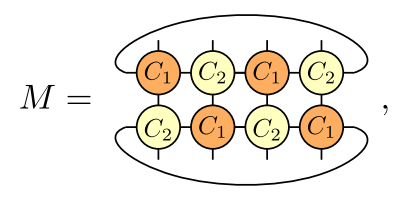

(a)

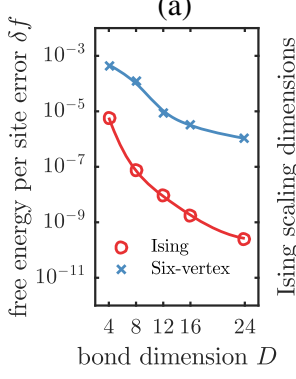

(b)

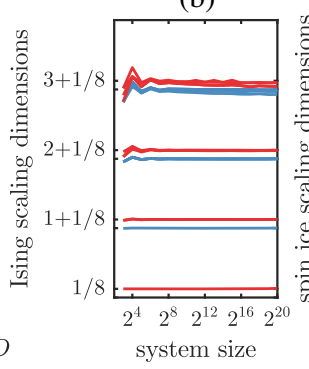

(c)

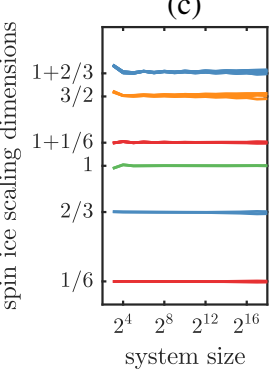

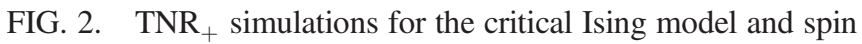
ice. (a) Relative error of the free energy per site in function of the $\mathrm{TNR}_{+}$bond dimension ( $N=2^{32}$ sites). (b),(c) Scaling dimensions extracted from the linear transfer matrix MPO Eq. (5) in function of the RG step (Ising $D=16$, spin ice $D=12$ ). 
TABLE I. Smallest scaling dimensions extracted from the eigenvalues of the radial transfer matrix MPO Eq. (3) for the critical Ising model (left) and spin ice (right).

\begin{tabular}{lccc}
\hline \hline Exact & Ising $\mathrm{TNR}_{+}(6)$ & Exact & Spin ice $\mathrm{TNR}_{+}(10)$ \\
\hline 0.125 & 0.125236 & $1 / 6$ & 0.163117 \\
1 & 0.999282 & $1 / 6$ & 0.167204 \\
1.125 & 1.123883 & $2 / 3$ & 0.659684 \\
1.125 & 1.123883 & $2 / 3$ & 0.662008 \\
2 & 1.998575 & 1 & 0.997413 \\
2 & 1.992823 & 1 & 0.997286 \\
2 & 1.996882 & $7 / 6$ & 1.163503 \\
2 & 1.994090 & $7 / 6$ & 1.163503 \\
\hline \hline
\end{tabular}

in a function of the system size (or, equivalently, iteration step) in Figs. 2(c) and 2(d) [15]. We observe that the numerically obtained implicit fixed point is stable under subsequent coarse-graining and remains so for a prolonged number of steps, in agreement with other TNR approaches $[8,12,32]$. To verify that the implicitly scale-invariant tensors are also explicitly approximately scale invariant after gauge fixing, we have constructed the radial transfer matrix MPO Eq. (3) and calculated its smallest scaling dimensions (see Table I). Together with the coarse-graining procedure described in Fig. 1, Eq. (3) can be used to study the fusion of primary fields and to calculate the operator product expansion coefficients of the underlying CFT, as has previously been done only using MERA-TNR for the Ising model [11]. More importantly, our results suggest that the characteristic information of the underlying CFT can also be obtained from the fixed point MPS tensors Eq. (4), which in our formalism act as transparent building blocks for both the linear and radial transfer matrix MPOs.

Effective Hamiltonians.-In Fig. 3, we have plotted nonnegative fixed point tensors [33] for the Ising model at $T<T_{c}, T=T_{c}$, and $T>T_{c}$. Because of the elementwise non-negativity, it is possible to equivalently consider the elementwise logarithm, so that we can interpret the tensor elements as energies of the configurations of the bonds connected to the site. The trivial tensor $C^{\text {triv }}$ for $T>T_{c}$ has one dominant element, and all other arbitrarily small

(a) $T=0.95 \cdot T_{c}$

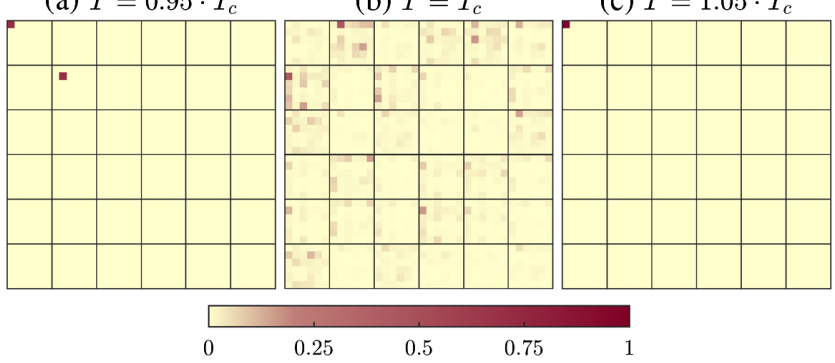

FIG. 3. Non-negative tensor elements of normalized fixed point tensors $C_{1, A}^{*}$ obtained from $D=6 \mathrm{TNR}_{+}$simulations of the Ising model at (a) $T<T_{c}$, (b) $T=T_{c}$, and (c) $T>T_{c}$. elements can be regarded as penalty terms in the effective Hamiltonian, signifying the use of a superfluous bond dimension in the description of the numerical fixed point. Similarly, for $T<T_{c}$, the $Z_{2}$ symmetry-breaking tensor $C^{\mathrm{Z}_{2}}=C^{\text {triv }} \oplus C^{\text {triv }}$ is given by two equal dominant values with all other elements effectively zero. Both of these fixed points satisfy the algebraic relations Eq. (4), since they are exact fixed points of the RG flow. Off-criticality we thus recover the fixed points previously found by $\mathrm{Gu}$ and Wen [34]. The critical fixed point tensor for $T=T_{c}$, however, is highly nontrivial, implying that the MPS optimization explores the full parameter space to approximate the exact fixed point which has an infinite bond dimension. Because of the lattice geometry and the choice of the local coarse-graining transformation, the effective Hamiltonian encoded in the critical fixed point is given by local interactions between at most four effective $D$-dimensional degrees of freedom $[35,36]$. Note that the MPS tensors encoded in the critical fixed point, part of which is shown in Fig. 3(b), provide an explicit and nontrivial example of numerically optimized solutions which approximately satisfy the algebraic fixed point equations Eq. (4) of the $\mathrm{TNR}_{+}$flow.

Conclusion and outlook.-We have proposed a manifestly non-negative tensor network renormalization algorithm to coarse-grain classical partition functions in real space and provided additional evidence that tensor network renormalization techniques provide an approximation that behaves in a controlled way, introducing the required freedom to approximate the relevant physics at larger length scales using effective interactions among effective degrees of freedom that are determined variationally. By restricting to non-negative tensors, our work provides a bridge between heuristic block-spin prescriptions and modern tensor network approaches to coarsegraining.

Further improvement of the numerical results should be possible by taking lattice and internal symmetries into account and by improving the control on the gauge freedom. Because of the algorithm's formulation in terms of periodic MPS, we expect that the interplay with wellestablished theoretical and numerical MPS and MPO results will be of great importance in this regard. A generalization of our scheme to the quantum case is possible by constructing sequences of completely positive maps acting on projected-entangled pair states wave functions [37]. Another application would be to incorporate the formalism of MPO algebras [38] in order to put topological restrictions on the CFT data extracted from tensor network renormalization $[39,40]$.

M. B. thanks L. Vanderstraeten, D. Williamson, and S. Yang for discussions. This work is supported by an Odysseus grant from the Fonds Wetenschappelijk Onderzoek (FWO), a PostDoc grant from the FWO (J. H.), European Research Council (ERC) grants 
Quantum Tensor Networks and Entanglement (QUTE) and Entanglement and Renormalisation for Quantum Fields (ERQUAF), and the EU grant Simulators and Interfaces with Quantum Systems (SIQS).

[1] L. P. Kadanoff, Physics 2, 263 (1966); Phys. Rev. Lett. 34, 1005 (1975); L. P. Kadanoff, A. Houghton, and M. C. Yalabik, J. Stat. Phys. 14, 171 (1976).

[2] K. G. Wilson, Rev. Mod. Phys. 47, 773 (1975).

[3] M. Levin and C. P. Nave, Phys. Rev. Lett. 99, 120601 (2007).

[4] Z.-C. Gu, M. Levin, and X.-G. Wen, Phys. Rev. B 78, 205116 (2008).

[5] Z. Y. Xie, H. C. Jiang, Q. N. Chen, Z. Y. Weng, and T. Xiang, Phys. Rev. Lett. 103, 160601 (2009).

[6] H. H. Zhao, Z. Y. Xie, Q. N. Chen, Z. C. Wei, J. W. Cai, and T. Xiang, Phys. Rev. B 81, 174411 (2010).

[7] Z. Y. Xie, J. Chen, M. P. Qin, J. W. Zhu, L. P. Yang, and T. Xiang, Phys. Rev. B 86, 045139 (2012).

[8] G. Evenbly and G. Vidal, Phys. Rev. Lett. 115, 180405 (2015).

[9] G. Evenbly and G. Vidal, Phys. Rev. Lett. 115, 200401 (2015).

[10] G. Evenbly, Phys. Rev. B 95, 045117 (2017).

[11] G. Evenbly and G. Vidal, Phys. Rev. Lett. 116, 040401 (2016).

[12] S. Yang, Z.-C. Gu, and X.-G. Wen, Phys. Rev. Lett. 118, 110504 (2017).

[13] "[...] the more recent tensor-style work often employs indices which are summed over hundreds of values, each representing a sum of configurations of multiple spinlike variables. All these indices are generated and picked by the computer. The analyst does not and cannot keep track of the meaning of all these variables. Therefore, even if a fixed point were generated, it would not be very meaningful to the analyst. In fact, the literature does not seem to contain much information about the values and consequences of fixed points for the new style of renormalization" [14].

[14] E. Efrati, Z. Wang, A. Kolan, and L. P. Kadanoff, Rev. Mod. Phys. 86, 647 (2014).

[15] See Supplemental Material at http://link.aps.org/ supplemental/10.1103/PhysRevLett.118.250602 for details on non-negative matrix factorization, numerical implementations, entanglement filtering, symmetries, approximate scale invariance, and extracting conformal data from tensor networks, which includes Refs. [16-26].

[16] N. Gillis, J. Mach. Learn. Res. 13, 3349 (2012).

[17] D. Donoho and V. Stodden, Proc. Adv. Neural Inf. Process. Syst. 16, 1141 (2004).

[18] K. Huang, N. D. Sidiropoulos, and A. Swami, IEEE Trans. Signal Process. 62, 211 (2014).

[19] S. A. Vavasis, SIAM J. Optim. 20, 1364 (2010).

[20] H. S. Seung and D. D. Lee, Nature (London) 401, 788 (1999).
[21] Y.-X. Wang and Y.-J. Zhang, IEEE Trans. Knowl. Data Eng. 25, 1336 (2013).

[22] N. Gillis, in Regularization, Optimization, Kernels, and Support Vector Machines, edited by M. S. J. A. K. Suykens and A. Argyriou (Chapman and Hall/CRC, London, 2014).

[23] C. Boutsidis and E. Gallopoulos, Pattern Recognition 41, 1350 (2008).

[24] P. A. Knight, SIAM J. Matrix Anal. Appl. 30, 261 (2008).

[25] D. Pérez-García, M. M. Wolf, M. Sanz, F. Verstraete, and J. I. Cirac, Phys. Rev. Lett. 100, 167202 (2008).

[26] J. Cirac, D. Prez-Garca, N. Schuch, and F. Verstraete, Ann. Phys. (Amsterdam) 378, 100 (2017).

[27] Given the nature of the problem, one might expect an $l_{1}$ norm. In practice, tackling the $l_{1}$-norm optimization problem is not economical due to the large number of constraints, hence the relaxation to a smooth optimization in practice.

[28] Note that all these boundary tensor networks are but different low-rank tensor network approximations of the leading eigenvector of the transfer matrix written as a matrix product operator (MPO) [29].

[29] J. Haegeman and F. Verstraete, Annu. Rev. Condens. Matter Phys. 8, 355 (2017).

[30] Note that, by working with symmetric tensor networks, we can also extract CFT data of nonlocal fields if we modify Eq. (3) to include a MPO threading through the transfer matrix (which encodes the antiperiodic boundary conditions and reduces to just a string of matrices for the tensor product symmetry considered in Ref. [11]). Similarly, the algebraic fixed point equations (4) can be modified to include an additional MPO symmetry string.

[31] M. Bal et al. (unpublished).

[32] Eventually, though, the accumulated truncation errors act as a relevant perturbation steering the flow away from criticality.

[33] We have plotted only $C_{1, A}^{*}$, but the behavior of the other tensors $C_{2, A}^{*}, C_{1, B}^{*}$, and $C_{2, B}^{*}$ is very similar. See also Appendix V [15].

[34] Z.-C. C. Gu and X.-G. G. Wen, Phys. Rev. B 80, 155131 (2009).

[35] A comprehensive analysis of the nature of these effective Hamiltonians will be reported elsewhere [31].

[36] Although one might be tempted to extend the domain of the coarse-graining operation to even bigger blocks, there is of course a numerical trade-off between the locality of a coarse-graining scheme and the bond dimension that can be attained in practice.

[37] F. Verstraete and J. I. Cirac, arXiv:cond-mat/0407066.

[38] N. Bultinck, M. Mariën, D. Williamson, M. Şahinoğlu, J. Haegeman, and F. Verstraete, Ann. Phys. (Amsterdam) 378, 183 (2017).

[39] D. Aasen, R. S. K. Mong, and P. Fendley, J. Phys. A 49, 354001 (2016).

[40] M. Hauru, G. Evenbly, W. W. Ho, D. Gaiotto, and G. Vidal, Phys. Rev. B 94, 115125 (2016). 